\title{
Double optimal density gradients for harmonic generation from relativistically oscillating plasma surfaces
}

Cite as: Phys. Plasmas 26, 103102 (2019); doi: 10.1063/1.5097440

Submitted: 26 March 2019 - Accepted: 12 September 2019 •

Published Online: 7 October 2019

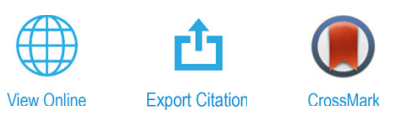

\author{
Jian Gao, ${ }^{1,2, a)}$ iD Boyuan Li, ${ }^{1,2, a)}$ iD Feng Liu, ${ }^{1,2, b)}$ Huaipeng Cai, ${ }^{1,2}$ Min Chen, $^{1,2}$ (D) Xiaohui Yuan, ${ }^{1,2}$ Xulei Ge, $^{1,2}$ \\ Liming Chen, ${ }^{1,2}$ Zhengming Sheng, ${ }^{1,2,3,4}$ and Jie Zhang ${ }^{1,2, c)}$
}

\author{
AFFILIATIONS \\ ${ }^{7}$ Key Laboratory for Laser Plasmas (Ministry of Education) and School of Physics and Astronomy, Shanghai Jiao Tong University, \\ Shanghai 200240, China \\ ${ }^{2}$ Collaborative Innovation Center of IFSA (CICIFSA), Shanghai Jiao Tong University, Shanghai 200240, China \\ ${ }^{3}$ SUPA, Department of Physics, University of Strathclyde, Glasgow G4 ONG, United Kingdom \\ ${ }^{4}$ Tsung-Dao Lee Institute, Shanghai Jiao Tong University, Shanghai 200240, China

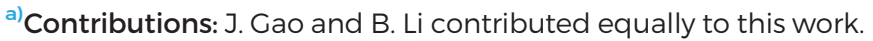

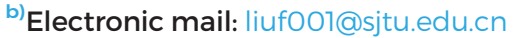 \\ ${ }^{c)}$ Electronic mail: jzhang1@sjtu.edu.cn
}

\begin{abstract}
The influence of plasma density gradients on the harmonic generation process from relativistically oscillating mirror (ROM) is studied experimentally. It is observed that the harmonic intensities from orders of 21st to 24th first drop and then reach the maximum value with the increase of plasma scale length $L .2 \mathrm{D}$ particle-in-cell simulations are performed to investigate the intensities of high-order harmonics at different $L$ values, which show that there are two optimal scale lengths $L_{o p t}$ for efficient ROM harmonic generation. The two optimal $L_{o p t}$ values are interpreted by a quasi-1D analytical model. By matching the potential energies provided by laser pulse and charge displacement in the plasma with an exponential distributed density profile, the model can quantitatively predict the optimal $L_{o p t}$ at the given laser incidence angle and intensity. Our work is beneficial for better understanding the role of $L$ in ROM harmonic generation and the parameters affecting the optimal $L_{\text {opt }}$.
\end{abstract}

(C) 2019 Author(s). All article content, except where otherwise noted, is licensed under a Creative Commons Attribution (CC BY) license (http:// creativecommons.org/licenses/by/4.0/). https://doi.org/10.1063/1.5097440

\section{INTRODUCTION}

High-order harmonic generation (HHG) by reflection of intense laser pulses from overdense plasma surfaces has been a subject of considerable interest in recent decades. ${ }^{1-3}$ The main motivation that drives such a field is to obtain coherent extreme ultraviolet (EUV) or $\mathrm{x}$-ray radiation. ${ }^{4,5}$ In the time domain, the superposition of harmonics leads to the generation of attosecond (as) pulse trains. ${ }^{6}$ Recently, it has been further extended to generation of single isolated attosecond pulse by various gating techniques. ${ }^{7-10}$ Owing to the near diffraction-limited beam quality ${ }^{11}$ with an attosecond (as) duration, it can be focused to extremely high intensity and applied in high energy density physics. ${ }^{12}$

In the relativistic regime, where the normalized vector potential $a_{0}=e E / m \omega_{0} c \geq 1$ ( $e$ and $m$ are the electron charge and mass, $E$ and $\omega_{0}$ are the amplitude and frequency of the laser pulse electric field, and $c$ is the light velocity in vacuum), the harmonic generation process can be described by the relativistically oscillating mirror (ROM) mechanism. ${ }^{1,13-16}$ In this mechanism, the plasma surface is driven to oscillate periodically by the laser pulse with velocity $v \sim c$. High order harmonics are generated when the incident pulse is reflected by this relativistically oscillating surface. According to the $\gamma$-spike model ${ }^{16}$ based on the ultrarelativistic similarity theory, ${ }^{17}$ the conversion efficiency $\eta$ of the ROM harmonics decays as $\eta \sim H^{-8 / 3}$ up to the cutoff frequency $\omega_{\text {cutoff }} \sim 8^{1 / 2} \gamma_{\max }^{3} \omega_{0}$. Here, $H$ is the harmonic order and $\gamma_{\max }$ is the maximum Lorentz factor of the plasma surface.

A crucial parameter for HHG from the ROM mechanism is the steepness of the density gradient at the plasma-vacuum interface. In the theoretical model, ${ }^{1,13-16}$ the plasma density profile is treated as a steplike function. In experiment, the harmonic emission is 
independent of the prepulse level over a wide range for harmonics generated by picosecond (ps) laser pulses. ${ }^{18,19}$ This suggests that the ponderomotive pressure has enough time to push the plasma inward to form a sharp density gradient. As the laser pulse duration is reduced to the femtosecond (fs) range, the efficiency of HHG is shown to be very sensitive to the laser contrast, due to the fact that the density gradient of plasma has insufficient time to be modified by the main pulse itself. Therefore, the laser contrast must be controlled when the main pulse arrives. To acquire a steplike density profile, a plasma mirror (PM) system is usually adopted to improve the laser contrast. ${ }^{20,21}$ Recent results show that a step-like density profile is not beneficial for the ROM harmonic generation and a steep density gradient with scale length $L$ in the range of $0<L<\lambda$ is preferred, ${ }^{22-27}$ where $\lambda$ is the laser wavelength. Dollar et al. proved that the generation efficiency and spectral structure of ROM harmonics are optimized around $L=c / \omega_{0}$ in experiments and simulations. ${ }^{26}$ Kahaly et al. continuously tuned $L$ in the range of $0<L<0.15 \lambda$ to investigate the role of $L$ experimentally. ${ }^{27}$ They demonstrate that there is an optimal scale length $L_{\text {opt }}$ in the range of $0.05 \lambda<L<0.1 \lambda$ for the ROM harmonics. The influence of $L$ on ROM harmonics can be understood qualitatively. If $L$ is too short, the restoring force of ions is so large that the electrons cannot obtain enough energies. With longer $L$, the restoring force becomes weaker. The momenta of outgoing electrons become larger, and the ROM harmonics can be generated efficiently. If $L$ is further increased, the thickness of the electron sheet at the plasma surface becomes large. The surface cannot be regarded as a mirror, which makes the emission incoherent. Thus, there is an optimal $L_{o p t}$ for efficient ROM harmonic generation.

In this article, we experimentally investigate the effect of the plasma density gradient on harmonic generation dominated by the ROM mechanism. Different from the results mentioned above, we find that with $L$ increasing, the harmonic intensities from orders of 21 st to 24 th first decrease to reach a minimal value and then increase to the maximum value. $2 \mathrm{D}$ particle-in-cell (PIC) simulations are also carried out. In simulations, two optimal $L_{\text {opt }}$ values are obtained; one is shorter than $0.1 \lambda$, and the other is longer than $0.1 \lambda$. The upper optimal $L_{o p t}$ is very close to the value observed in our experiments. The experimental and simulation results are explained by a quasi-1D analytical model. By matching the potential energies provided by laser pulse and charge displacement in the plasma with an exponential distributed density profile, the two optimal $L_{\text {opt }}$ values can be quantitatively estimated for efficient ROM harmonic generation at the given laser incidence angle and intensity.

\section{EXPERIMENTS}

The experimental setup is shown in Fig. 1. We use the 200 TW Ti:sapphire laser system $(800 \mathrm{~nm}, 29 \mathrm{fs})$ at the Laboratory for Laser Plasmas of Shanghai Jiao Tong University. The laser temporal contrast is improved to be better than $\sim 10^{-11}$ at 10 ps prior to the main peak by combining a single plasma mirror (PM) system $^{28}$ and a crosspolarized wave (XPW) generation technique. ${ }^{29}$ The p-polarized laser beam with an energy of $460 \mathrm{~mJ}$ is used in the experiments and focused onto polished fused silica plates by an $f / 4$ off-axis parabola (OAP) mirror with a focal spot diameter of $6 \mu \mathrm{m}$ (full width at half maximum, FWHM). The calculated peak intensity of the laser pulse on the target is $I=1.9 \times 10^{19} \mathrm{~W} / \mathrm{cm}^{2}\left(a_{0}=3\right)$. The incidence angle of the

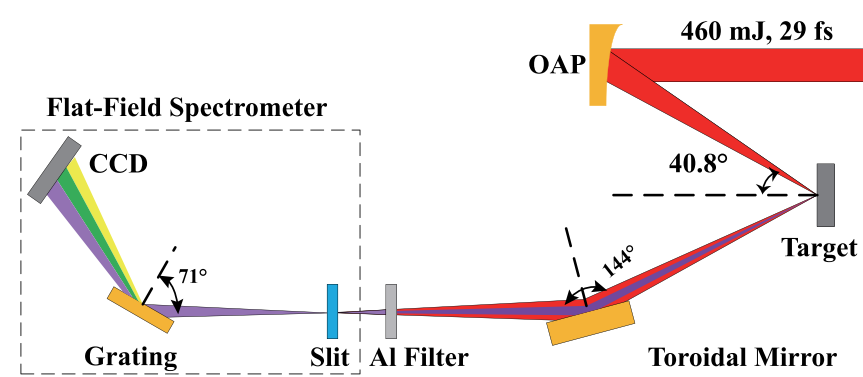

FIG. 1. The sketch of the experimental setup. A gold coated toroidal mirror is used to focus the harmonics to a flat-field grazing incidence spectrometer with a toroidal grating $(290$ lines $/ \mathrm{mm})$. A $250 \mathrm{~nm}$ thick aluminum filter is placed in front of the spectrometer to block the laser beam.

laser beam is $40.8^{\circ}$, and the high-order harmonic radiation is measured using a flat-field spectrometer.

In order to control $L$ when the main pulse arrives, a prepulse with the same duration as the main pulse and peak intensity of $I=3.2 \times 10^{17} \mathrm{~W} / \mathrm{cm}^{2}\left(a_{0}=0.4\right)$ is introduced to ionize the target. $L$ could be continuously controlled by adjusting the delay $T$ between the prepulse and the main pulse. ${ }^{27}$ 1D PIC simulations with mobile ions are utilized to calculate the temporal evolution of the plasma density profile at the target surface after prepulse irradiation, so that $L$ at different prepulse delays $T$ from 0 ps to 5 ps could be obtained.

The influence of $L$ on harmonics of the coherent wake emission (CWE) mechanism ${ }^{30}$ was investigated experimentally and numerically in our previous research. ${ }^{31}$ It was found that there is an optimal $L_{o p t}$ for CWE harmonics around $L=0.1 \lambda$. In this article, we concentrate on the effect of $L$ on ROM harmonics $(H>20$ th). The typical raw image (top panel) and its integrated harmonic spectrum (bottom panel) are shown in Fig. 2(a) at $L=0.17 \lambda$. The integrated harmonic intensities from orders of 21st to 24th as a function of $L$ (bottom axis) and the corresponding $T$ (top axis) are plotted in Fig. 2(b). It can be clearly seen that when $L$ increases from $0.08 \lambda$ to $0.36 \lambda$, the harmonic intensities first drop to reach a minimal value at $L=0.11 \lambda$. Then, the intensities increase to the highest value at $L=0.17 \lambda$. When $L>0.17 \lambda$, the intensities decrease again.

\section{2D PIC SIMULATIONS}

In order to understand the experimental results, 2D PIC simulations are carried out. The size of the simulation box is $6 \lambda$ ( $x$ axis) $\times 18 \lambda$ (y axis). The plasma density is exponentially increasing along the $\mathrm{x}$ axis until it reaches the maximum of $100 n_{c}$. Then, the plasma density remains constant with a thickness of $2 \lambda(\lambda=800 \mathrm{~nm})$, which occupies $x>0$. The number of particles per cell is 144 . A p-polarized Gaussian beam with a duration of $10 T_{0}$ (FWHM) and a waist of $4 \lambda$ $(1 / e)$ obliquely irradiates the plasma at $\theta=40^{\circ}$ with respect to the $\mathrm{x}$ axis, where $T_{0}=\lambda / c$ is the period. The normalized vector potential of the laser pulse is $a_{0}=3$. The grid size of the simulation box is $d x$ $=d y=\lambda / 100$, and the time step is $d t=T_{0} / 200$. Using 2D Fourier transform, harmonics in the reflected direction are diagnosed.

Figure 3(a) shows the simulated harmonic intensity of each order with different $L$ values. In order to investigate the ROM harmonics, the integrated harmonic intensities from orders of 15th to 30th at different $L$ values are plotted in Fig. 3(b). Two optimal $L_{\text {opt }}$ values can be found. The harmonic intensities first increase rapidly with $L$ to reach a 

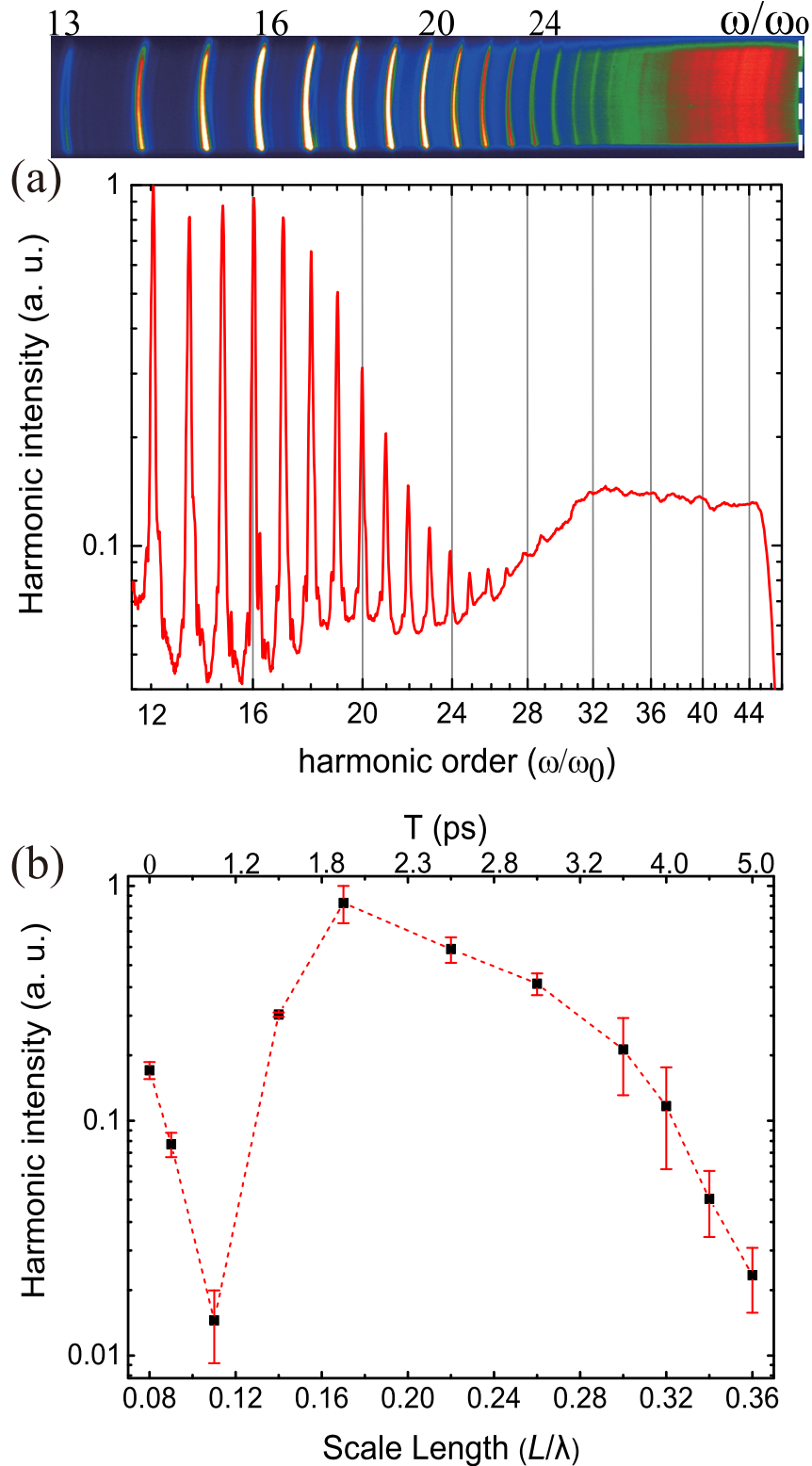

FIG. 2. (a) A typical raw image (top panel) and its integrated high-order harmonic spectrum (bottom panel) obtained at $a_{0}=3$ and $40.8^{\circ}$. The white dashed line indicates the aluminum absorption edge. (b) The dependence of integrated harmonic intensities from orders of 21st to 24th on preplasma scale length $L$ (bottom axis) and pulse delay $T$ (top axis) from experiments. The error bars show the standard deviation. The dashed line is drawn as a guide to the eye.

maximum at $L=0.07 \lambda$. After declining to a minimal value at $L=0.1 \lambda$, the harmonic intensities increase again to a second peak at $L=0.2 \lambda$. The evolution of harmonic intensities with $L$ is the same as that observed in experiments. Since the rising edge of our laser pulse inevitably produces preplasma before the main peak arrives even without introducing a prepulse, it prevents us observing the lower optimal $L_{\text {opt }}^{l}$ shorter than $0.1 \lambda$ in the experiments. Recently, Kormin et al. ${ }^{32}$ (a)
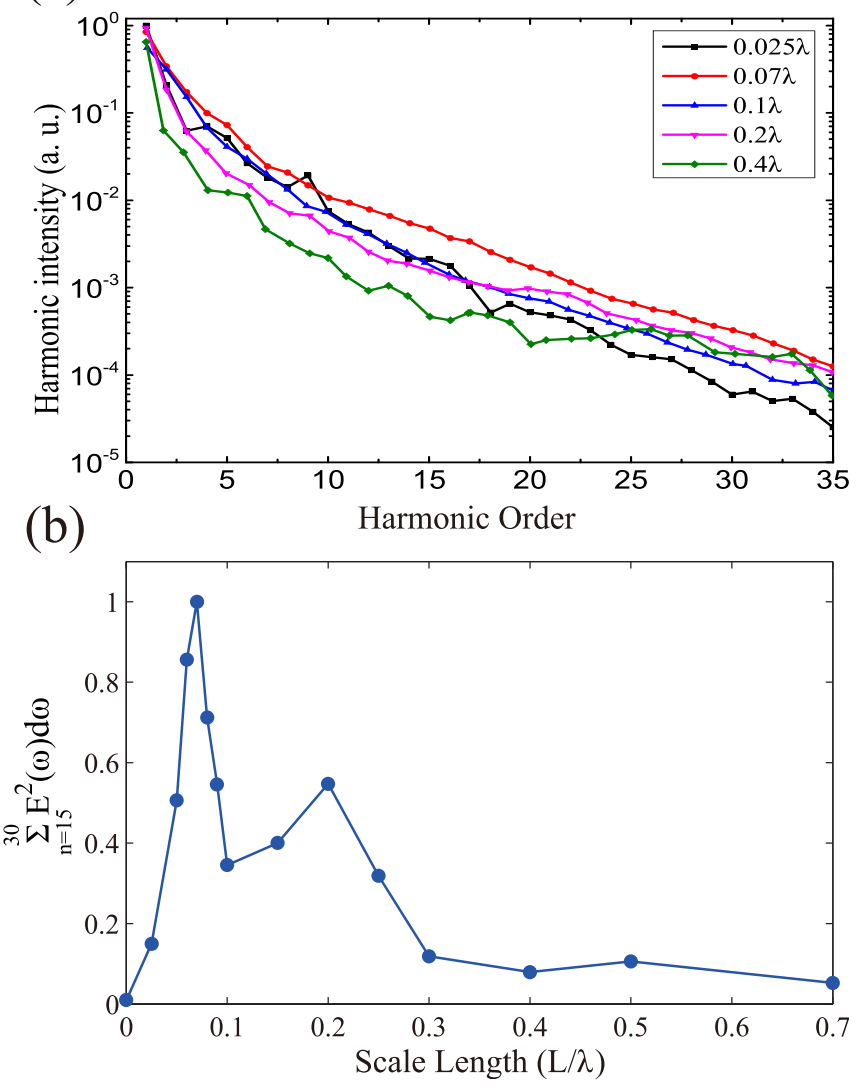

FIG. 3. Influence of $L$ on high-order harmonic emission dominated by the ROM mechanism from 2D PIC simulations. (a) The simulated harmonic intensity of each order with different $L$ values. (b) Relative integrated harmonic intensities $\Sigma E^{2}(\omega) d \omega$ (15th $\sim 30$ th) with different $L$ values. The solid lines in $(a)$ and $(b)$ are drawn as guides to the eye.

and Chopineau et al. ${ }^{33}$ showed a similar dependence of harmonic intensities on $L$.

\section{THEORETICAL MODEL}

In order to quantitatively explain the role of $L$ in the ROM harmonic generation process and estimate the value of the optimal $L_{o p t}$, a quasi-1D analytical model is developed. In the ROM scenario, electrons near the critical surface are first pushed forward by the ultrashort, relativistic laser pulse and build a restoring force with the immobile ions. At a later half cycle, the laser field direction is reversed and, consequently, both the restoring force and the laser field pull the electrons back. The backward electrons are strongly relativistic. When the velocity of these electrons at the plasma surface approaches $c$ and the $\gamma$ factor of the surface has a sharp peak, high-order harmonics are generated as the laser pulse is reflected by the relativistic electrons.

Let us consider in the laboratory frame $(\mathrm{L})$ that a p-polarized laser pulse with an incidence angle of $\theta$ irradiates a solid-density plasma with an exponential gradient density. The laser pulse is polarized in the $\mathrm{x}-\mathrm{y}$ plane, and $\mathrm{x}$ is the target normal direction. For simplification of analysis, we use a boosted frame $(\mathrm{M})^{14}$ to transform the laser 
pulse into normal incidence. The boosted frame moves along the $y$ direction with a velocity of $c \sin \theta$. After Lorentz transformation, one can find the following relations:

$$
\begin{gathered}
E_{y}^{M}=E^{L} \cos \theta, \quad B_{z}^{M}=B^{L} \cos \theta, \quad \omega_{0}^{M}=\omega_{0}^{L} \cos \theta, \\
a_{0}^{M}=a_{0}^{L}, \quad \gamma^{M}=1 / \cos \theta, \quad n^{M}=\gamma^{M} n^{L},
\end{gathered}
$$

where $B$ is the magnetic field of the laser pulse, $\gamma$ is the Lorentz factor, and $n$ is the plasma density. For convenience, the superscript $\mathrm{M}$ will be omitted. The canonical momentum of electrons in the field is conserved,

$$
\boldsymbol{p}_{y}-\boldsymbol{a}=\boldsymbol{p}_{y}^{0}, \quad \gamma-\boldsymbol{p}_{x}=\gamma^{0},
$$

where $\boldsymbol{p}_{\boldsymbol{y}}=\gamma \boldsymbol{\beta}_{y}$ and $\boldsymbol{p}_{\boldsymbol{x}}=\gamma \boldsymbol{\beta}_{x}$ are the transverse and longitudinal momentum normalized by $m c$ and $\boldsymbol{p}_{y}^{\mathbf{0}}$ and $\gamma^{0}$ are the momentum and Lorentz factor before the laser pulse arrives. From Eq. (1), we have $p_{y}^{0}=-\hat{y} \tan \theta$ and $\gamma^{0}=1 / \cos \theta$. The longitudinal momentum can be derived from Eq. (2), using $\gamma=\left(1+p_{x}^{2}+p_{y}^{2}\right)^{1 / 2}$,

$$
p_{x}=\frac{a^{2}}{2} \cos \theta+a \sin \theta,
$$

where $a=a_{0} \cos (\omega t)$. In the relativistic domain, the potential energy $\Phi_{L}$ that the laser exerts on electrons approximates to $\Phi_{L}=\overline{p_{x}} m c^{2}$. Therefore, averaging Eq. (3) in the pushing (or pulling) half cycle, $\Phi_{L}$ is

$$
\Phi_{L}^{ \pm}=\left(\frac{a_{0}^{2}}{4} \cos \theta \pm \frac{2}{\pi} a_{0} \sin \theta\right) m c^{2},
$$

where "+" represents the pushing phase and "-" represents the pulling phase. It is clear that the first term in Eq. (4) is the ponderomotive potential energy in the longitudinal direction, while the second term is the electric potential energy.

On the other hand, the potential energy $\Phi_{R}$ generated by charge displacement in plasma is built up at the critical surface during the electron oscillation. The plasma density $n$ at the critical surface satisfies $\omega_{0}^{2}=e^{2} n / \varepsilon_{0} m$ in the boosted frame. After transforming back to the laboratory frame, the position is determined by $n=n_{c} \cos ^{2} \theta$, where $n_{c}$ is the critical density in the laboratory frame. If we define this point as $x=0$, the density profile of the plasma is $n(x)=n_{c} \cos ^{2} \theta \exp (x / L)$. Due to the push-out of electrons, $\Phi_{R}$ is given by the Poisson equation $-\nabla^{2} \Phi_{R}=-e^{2} n / \varepsilon_{0}$. Integrating the $1 \mathrm{D}$ plasma density profile, $\Phi_{R}$ is

$$
\Phi_{R}(x)=\frac{e n_{c}}{\epsilon_{0}} L^{2} \cos ^{2} \theta \exp \left(\frac{x}{L}\right) .
$$

In the pushing phase, the maximum longitudinal excursion of relativistic electrons is $x_{s}=v_{x} / \omega_{0} \cos \theta \sim c / \omega_{0}$, where $v_{x}$ is the typical quivering velocity of electrons in the field. If $\Phi_{R}=\Phi_{L}^{+}$at this point, electrons will be pulled back with maximum energy in the next pulling phase. Hence, the generation of high-order harmonics reaches the optimum.

We define

$$
\Gamma=\frac{\Phi_{R}\left(x_{s}\right)-\Phi_{L}^{+}}{\Phi_{L}^{+}}
$$

as the dimensionless matching parameter between $\Phi_{L}$ and $\Phi_{R}$. There are three cases, as shown in Fig. 4(a). (i) $\Gamma=0 . \Phi_{L}$ is exactly (a)

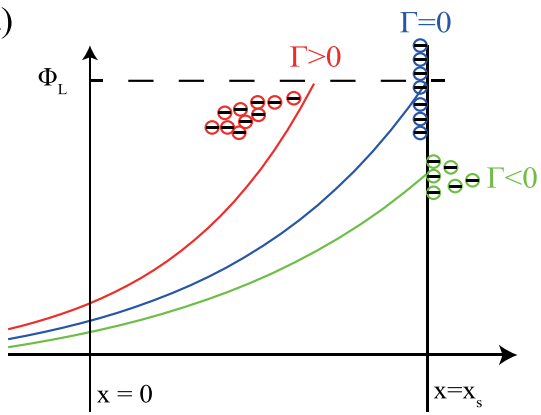

(b)

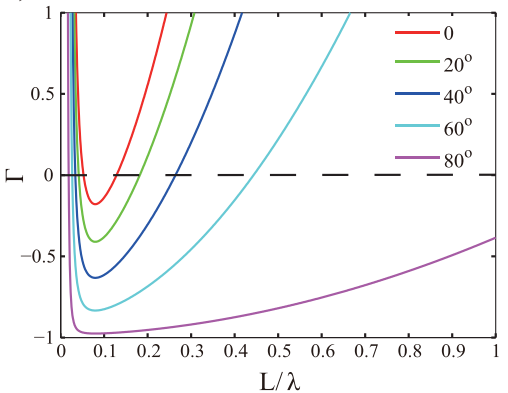

FIG. 4. (a) Sketch of the three cases of matching parameter $\Gamma$. The colored lines represent $\Phi_{R}(x)$, and the balls represent electrons. (b) $\Gamma$ as a function of $L$ for different incidence angles calculated by the theoretical model at $a_{0}=3$.

transformed to $\Phi_{R}$, and a thin sheet of electrons is formed at $x=x_{s}$. Later, these electrons gain the maximum $\gamma$ and radiate harmonics coherently in the pulling phase. (ii) $\Gamma<0$. Although the local electrons at $x=x_{s}$ could be pulled back by $\Phi_{R}$, the electron momentum is relatively low and the harmonic emission is weak. (iii) $\Gamma>0$. Electrons will be bounced back by $\Phi_{R}$ before they reach $x_{s}$. The electrons will be out of phase with the field after a few cycles of oscillation. Therefore, electrons cannot be stacked into a thin sheet, destroying the coherence of high-order harmonics.

Figure 4(b) shows the dependence of $\Gamma$ on $L$ for different incidences angles. From this quasi-1D model, we can estimate the optimal $L_{\text {opt }}$ at the given incidence angle and intensity of the laser pulse. The points at where $\Gamma=0$ correspond to the optimal $L_{\text {opt }}$ for a certain incidence angle at $a_{0}=3$. As shown in Fig. 4(b), two optimal $L_{\text {opt }}$ values can be found for efficient harmonic generation, the lower value of $L_{\text {opt }}^{l}<0.1 \lambda$ and the upper value of $L_{o p t}^{u}>0.1 \lambda$. Between these two optimal $L_{o p t}$, the harmonic intensity first decreases and then increases. Around $L=0.1 \lambda$, the harmonic intensity is minimal. Upon increasing the incidence angle, the lower value of $L_{\text {opt }}^{l}$ decreases and the upper value of $L_{o p t}^{u}$ increases. If the incidence angle is $80^{\circ}$, the upper optimal $L_{o p t}^{u}$ will be much larger than $\lambda$. The electron sheet thickness would be too large, so that the radiation is incoherent. Therefore, there is only one optimal $L_{\text {opt }}$ in the range of $0<L<\lambda$. In addition, from the model, it can also be derived that with increasing $a_{0}$, the value of $L_{o p t}^{l}$ decreases and the value of $L_{o p t}^{u}$ increases. The optimal $L_{o p t}=0.17 \lambda$ is found in our experiments, and the optimal $L_{o p t}^{u}=0.2 \lambda$ is found in simulations. These two optimal values of $L_{\text {opt }}$ are very close to the optimal $L_{\text {opt }}^{u}=0.26 \lambda$ estimated by the quasi-1D model in Fig. 4(b). 


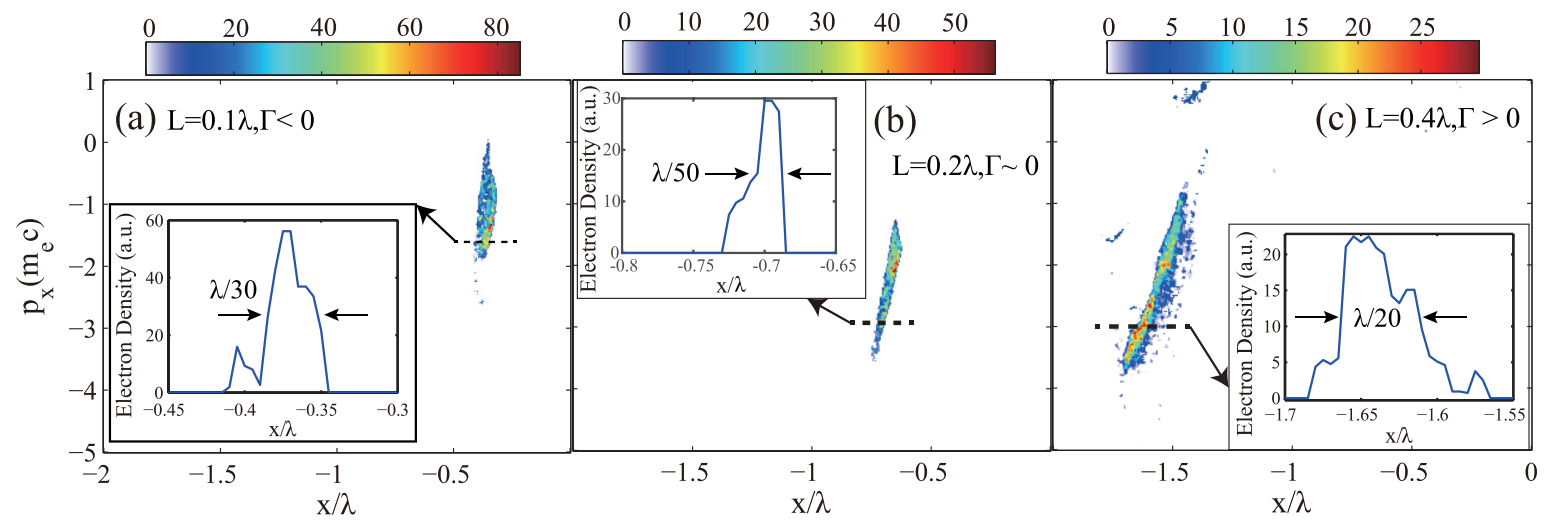

FIG. 5. (a), (b), and (c) Electron density distributions $n_{e}\left(x, p_{x}\right)$ in the phase space obtained from $2 \mathrm{D}$ PIC simulations for $L=0.1 \lambda, L=0.2 \lambda$, and $L=0.4 \lambda$, respectively. The insets in (a), (b), and (c) show the density profiles along the dashed line in each figure.

Physically, there are two optimal $L_{\text {opt }}$ values for the ROM harmonic generation because the efficiency can be improved by increasing either the radiation power of single electron or the number of electrons contributed to the radiation. According to the radiation theory, ${ }^{34}$ the emission amplitude $E_{H} \propto N \gamma \dot{p}$, where $N$ is the local electron number, $\gamma$ represents the net energy of electrons gained from the laser pulse, and $\dot{p}$ is the change rate of electron momentum depending on the laser intensity and incidence angle. The electron sheet has low $\gamma$ but large electron number $N$ at shorter $L_{\text {opt }}^{l}$. On the other hand, the electron sheet has very large $\gamma$ but low electron number $N$ at longer $L_{o p t}^{u}$.

Actually, in our model, we regard $\Phi_{L}$ being exactly transformed to $\Phi_{R}$ (matching parameter $\Gamma=0$ ) as the maximum harmonic generation efficiency for ROM. All the oscillating electrons are assumed to obtain the maximum energy, regardless of the longitudinal profile of electrons. Therefore, the value of the optimal $L_{o p t}$ will be closer to the model when the higher order harmonics are integrated.

To further illustrate the influence of $L$ on ROM harmonics, the electron density distributions $n_{e}$ in the phase space $\left(x-p_{x}\right)$ when the velocity of electrons is maximum in the pulling phase from the $2 \mathrm{D}$ PIC simulations are analyzed in Figs. 5(a)-5(c). The electrons with $\gamma \geq 2$ and along the central normal of the target are selected. To get enough number of electrons for statistics, the electrons falling in an ultrathin stripe with $\delta y=\lambda / 20$ are counted. In Figs. 5(a) and 5(b), $\Gamma \leq 0$, the electrons are bunched backward and stacked into a thin sheet. However, in Fig. 5(c), $\Gamma>0$, the electrons are relatively dispersed. The electron layer is not flat; meanwhile, electrons moving forward can also be seen in the figure. The electron distributions agree with the descriptions in Fig. 4(a). As our theoretical analysis, the important merit of $\Gamma=0$ for HHG is the enhancement on radiation coherence. The electron density profiles of the most energetic electrons are shown in the insets in Figs. 5(a)-5(c). The positions of electron profiles are chosen at $p_{x}=-1.8$ in Fig. 5(a) and $p_{x}=-3$ in Figs. 5(b) and $5(\mathrm{c})$. The beam thickness is $\lambda / 30$ for Fig. $5(\mathrm{a}), \lambda / 50$ for Fig. 5(b), and $\lambda / 20$ for Fig. 5(c). It means that even the 50th harmonics can be coherently generated at $\Gamma \sim 0$. However, the coherent emission of high-order harmonics is limited at $\Gamma \neq 0$.

The shape of the harmonic spectrum is determined by the electron momentum distribution. Figure 5(a) shows a lower momentum of the electron beam compared to Fig. 5(b). Quantitatively, few electrons can be seen in the $\left|p_{x}\right| \geq 2\left(4 \gamma_{\max }^{2} \geq 16\right)$ region in Fig. 5(a), and so the harmonic intensities for $H>15$ are low. However, in the $\left|p_{x}\right|<2$ region, the electron density in Fig. 5(a) is larger, which can produce stronger harmonics for $H<15$, as shown in Fig. 3(a). In Fig. $5(\mathrm{c})$, the beam thickness is reduced around $\left|p_{x}\right|=2.5\left(4 \gamma_{\max }^{2}=25\right)$ so that we can see an enhancement of $H=25 \sim 30$ harmonics on the $L=0.4 \lambda$ spectrum in Fig. 3(a). The local reduction of the beam thickness may be due to the dynamic coupling of $\Phi_{L}$ and $\Phi_{R}$, which is not included in our theoretical model.

\section{CONCLUSIONS}

In conclusion, the role of the plasma density gradient at the solid surface in the HHG by the ROM mechanism has been investigated experimentally. In experiments, at an incidence angle of $40.8^{\circ}$ and $a_{0}=3$, we obtain the optimal harmonic intensities at $L_{o p t}$ $=0.17 \lambda$ by integrated harmonic orders from 21 st to 24 th. Meanwhile, the harmonic intensities are minimal at $L_{o p t}=0.11 \lambda$. 2D PIC simulations are performed, which show that two optimal $L_{\text {opt }}$ values are found, one optimal value at $L_{\text {opt }}^{l}=0.07 \lambda$, while the other optimal value at $L_{o p t}^{u}=0.2 \lambda$. At $L=0.1 \lambda$, the harmonic intensities are minimal. The two optimal $L_{\text {opt }}$ values for efficient ROM harmonic generation are quantitatively interpreted by a quasi-1D analytical model, when the dimensionless parameter $\Gamma=0$ at the given incidence angle and intensity of laser pulses for an exponential distributed preplasma density profile. Although the shorter optimal $L_{\text {opt }}^{l}$ is not reached experimentally owing to the limited laser contrast, the expected tendency of ROM harmonic generation efficiency has been outlined. Our results are beneficial for a quantitative understanding of the role of $L$ in the ROM harmonic generation process, in which the value of the optimal $L_{o p t}^{l}$ is affected by the laser incidence angle and intensity.

\section{ACKNOWLEDGMENTS}

The research leading to these results received support from the National Basic Research Program of China (Grant No. 2013CBA01504), National Natural Science Foundation of China (Grant Nos. 11721091, 11305103, 11775144, and 11905129), sponsored by the Natural Science Foundation of Shanghai (Grant 
Nos. 18ZR1419200 and 13ZR1456300), and Project funded by the China Postdoctoral Science Foundation (Grant No. 2017M621443). Simulations were performed on the PI Supercomputer at SJTU.

\section{REFERENCES}

${ }^{1}$ P. Gibbon, Phys. Rev. Lett. 76, 50 (1996).

${ }^{2}$ C. Thaury and F. Quéré, J. Phys. B: At., Mol. Opt. Phys. 43, 213001 (2010).

${ }^{3}$ U. Teubner and P. Gibbon, Rev. Mod. Phys. 81, 445 (2009).

${ }^{4}$ B. Dromey, M. Zepf, A. Gopal, K. Lancaster, M. S. Wei, K. Krushelnick, M. Tatarakis, N. Vakakis, S. Moustaizis, R. Kodama, M. Tampo, C. Stoeckl, R. Clarke, H. Habara, D. Neely, S. Karsch, and P. Norreys, Nat. Phys. 2, 456 (2006).

${ }^{5}$ B. Dromey, S. Kar, C. Bellei, D. C. Carroll, R. J. Clarke, J. S. Green, S. Kneip, K. Markey, S. R. Nagel, P. T. Simpson, L. Willingale, P. McKenna, D. Neely, Z. Najmudin, K. Krushelnick, P. A. Norreys, and M. Zepf, Phys. Rev. Lett. 99, 085001 (2007).

${ }^{6}$ Y. Nomura, R. Hörlein, P. Tzallas, B. Dromey, S. Rykovanov, Z. Major, J. Osterhoff, S. Karsch, L. Veisz, M. Zepf, D. Charalambidis, F. Krausz, and G. D. Tsakiris, Nat. Phys. 5, 124 (2009).

${ }^{7}$ S. G. Rykovanov, M. Geissler, J. Meyer-ter-Vehn, and G. D. Tsakiris, New J. Phys. 10, 025025 (2008)

${ }^{8}$ M. Yeung, J. Bierbach, E. Eckner, S. Rykovanov, S. Kuschel, A. Sävert, M. Förster, C. Rödel, G. G. Paulus, S. Cousens, M. Coughlan, B. Dromey, and M. Zepf, Phys. Rev. Lett. 115, 193903 (2015).

${ }^{9}$ H. Vincenti and F. Quéré, Phys. Rev. Lett. 108, 113904 (2012).

${ }^{10} \mathrm{P}$. Heissler, R. Hörlein, J. M. Mikhailova, L. Waldecker, P. Tzallas, A. Buck, K. Schmid, C. M. S. Sears, F. Krausz, L. Veisz, M. Zepf, and G. D. Tsakiris, Phys. Rev. Lett. 108, 235003 (2012).

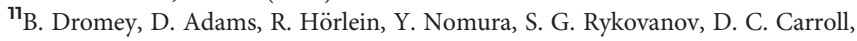
P. S. Foster, S. Kar, K. Markey, P. McKenna, D. Neely, M. Geissler, G. D. Tsakiris, and M. Zepf, Nat. Phys. 5, 146 (2009).

${ }^{12}$ S. Gordienko, A. Pukhov, O. Shorokhov, and T. Baeva, Phys. Rev. Lett. 94, 103903 (2005)

${ }^{13}$ S. V. Bulanov, N. M. Naumova, and F. Pegoraro, Phys. Plasmas 1, 745 (1994).

${ }^{14}$ R. Lichters, J. Meyer-ter-Vehn, and A. Pukhov, Phys. Plasmas 3, 3425 (1996).

${ }^{15} \mathrm{D}$. von der. Linde and K. Rzazewski, Appl. Phys. B: Lasers Opt. 63, 499 (1996).

${ }^{16}$ T. Baeva, S. Gordienko, and A. Pukhov, Phys. Rev. E 74, 046404 (2006).

${ }^{17}$ S. Gordienko and A. Pukhov, Phys. Plasmas 12, 043109 (2005).

${ }^{18}$ P. A. Norreys, M. Zepf, S. Moustaizis, A. P. Fews, J. Zhang, P. Lee, M. Bakarezos, C. N. Danson, A. Dyson, P. Gibbon, P. Loukakos, D. Neely,
F. N. Walsh, J. S. Wark, and A. E. Dangor, Phys. Rev. Lett. 76, 1832 (1996).

${ }^{19}$ J. Zhang, M. Zepf, P. A. Norreys, A. E. Dangor, M. Bakarezos, C. N. Danson, A. Dyson, A. P. Fews, P. Gibbon, M. H. Key, P. Lee, P. Loukakos, S. Moustaizis, D. Neely, F. N. Walsh, and J. S. Wark, Phys. Rev. A 54, 1597 (1996).

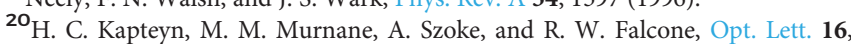
490 (1991)

${ }^{21}$ D. M. Gold, Opt. Lett. 19, 2006 (1994).

${ }^{22}$ M. Zepf, G. D. Tsakiris, G. Pretzler, I. Watts, D. M. Chambers, P. A. Norreys, U. Andiel, A. E. Dangor, K. Eidmann, C. Gahn, A. Machacek, J. S. Wark, and K. Witte, Phys. Rev. E 58, R5253 (1998).

${ }^{23}$ A. Tarasevitch, K. Lobov, C. Wünsche, and D. von der. Linde, Phys. Rev. Lett. 98, 103902 (2007).

${ }^{24}$ M. Behmke, D. an der Brügge, C. Rödel, M. Cerchez, D. Hemmers, M. Heyer, O. Jäckel, M. Kübel, G. G. Paulus, G. Pretzler, A. Pukhov, M. Toncian, T. Toncian, and O. Willi, Phys. Rev. Lett. 106, 185002 (2011).

${ }^{25}$ C. Rödel, D. an der Brügge, J. Bierbach, M. Yeung, T. Hahn, B. Dromey, S Herzer, S. Fuchs, A. G. Pour, E. Eckner, M. Behmke, M. Cerchez, O. Jäckel, D. Hemmers, T. Toncian, M. C. Kaluza, A. Belyanin, G. Pretzler, O. Willi, A. Pukhov, M. Zepf, and G. G. Paulus, Phys. Rev. Lett. 109, 125002 (2012).

${ }^{26}$ F. Dollar, P. Cummings, V. Chvykov, L. Willingale, M. Vargas, V. Yanovsky, C. Zulick, A. Maksimchuk, A. G. R. Thomas, and K. Krushelnick, Phys. Rev Lett. 110, 175002 (2013).

${ }^{27}$ S. Kahaly, S. Monchocé, H. Vincenti, T. Dzelzainis, B. Dromey, M. Zepf, P. Martin, and F. Quéré, Phys. Rev. Lett. 110, 175001 (2013).

${ }^{28}$ X. Ge, X. Yuan, Y. Fang, W. Wei, S. Yang, F. Liu, M. Chen, L. Zhao, Z. Sheng, and J. Zhang, Chin. Opt. Lett. 16, 103202 (2018).

${ }^{29}$ A. Jullien, O. Albert, F. Burgy, G. Hamoniaux, J.-P. Rousseau, J.-P. Chambaret, F. Augé-Rochereau, G. Chériaux, J. Etchepare, N. Minkovski, and S. M. Saltiel, Opt. Lett. 30, 920 (2005).

${ }^{30}$ F. Quéré, C. Thaury, P. Monot, S. Dobosz, P. Martin, J.-P. Geindre, and P. Audebert, Phys. Rev. Lett. 96, 125004 (2006).

${ }^{31}$ J. Gao, F. Liu, X. Ge, Y. Deng, G. Zhang, Y. Fang, W. Wei, S. Yang, X. Yuan, M. Chen, Z. Sheng, and J. Zhang, Chin. Opt. Lett. 15, 081902 (2017).

${ }^{32}$ D. Kormin, A. Borot, G. Ma, W. Dallari, B. Bergues, M. Aladi, I. B. Földes, and L. Veisz, Nat. Commun. 9, 4992 (2018).

${ }^{33}$ L. Chopineau, A. Leblanc, G. Blaclard, A. Denoeud, M. Thévenet, J.-L. Vay, G. Bonnaud, P. Martin, H. Vincenti, and F. Quéré, Phys. Rev. X 9, 011050 (2019).

${ }^{34}$ J. D. Jackson, Classical Electrodynamics, 3rd ed. (John Wiley \& Sons, New York, 1998). 